\title{
Collagen fleece-bound fibrin sealant is not associated with an increased risk of thromboembolic events or major bleeding after its use for haemostasis in surgery: a prospective multicentre surveillance study
}

\author{
Mathias Birth ${ }^{1}$, Joan Figueras ${ }^{2}$, Stéphane Bernardini ${ }^{3}$, Tine Troen ${ }^{4}$, \\ Klaus Günther ${ }^{5}$, Darius Mirza ${ }^{6}$ and Frank Viborg Mortensen*7
}

\begin{abstract}
Address: ${ }^{1}$ Klinik für Allgemein-, Viszeral-, Thorax- und Gefässchirurgie, HANSE-Klinikum Stralsund, Stralsund, Germany, ${ }^{2}$ Servicio de Cirugia General, Dr. Josep Trueta Hospital, IDiBGi, Girona, Spain, ${ }^{3}$ Service d'Urologie, CHU Saint Jacques, Besançon, France, ${ }^{4}$ Biostatistics, Nycomed, Roskilde, Denmark, ${ }^{5}$ Abteilung für Allgemein- und Viszeralchirurgie, Klinik Hallerwiese, Nürnberg, Germany, ${ }^{6}$ The Liver Unit, Queen Elizabeth Hospital and Birmingham Children's Hospital, Birmingham, UK and ${ }^{7}$ Kirurgisk Gastroenterologisk Afdeling L, Aarhus University Hospital, NBG, Aarhus, Denmark

Email: Mathias Birth - mathias.birth@klinikum-hst.de; Joan Figueras - info@jfigueras.net; Stéphane Bernardini - sbernardini@chu-besancon.fr; Tine Troen - Tine.Troen@nycomed.com; Klaus Günther - Klaus.Guenther@chir.med.uni-erlangen.de; Darius Mirza - Darius.Mirza@uhb.nhs.uk; Frank Viborg Mortensen* - fvmortensen@stofanet.dk

* Corresponding author
\end{abstract}

Published: 22 June 2009

Patient Safety in Surgery 2009, 3:13 doi:10.1 186/1754-9493-3-13

This article is available from: http://www.pssjournal.com/content/3/I/I3

(c) 2009 Birth et al; licensee BioMed Central Ltd.

This is an Open Access article distributed under the terms of the Creative Commons Attribution License (http://creativecommons.org/licenses/by/2.0), which permits unrestricted use, distribution, and reproduction in any medium, provided the original work is properly cited.
Received: 2 April 2009

Accepted: 22 June 2009

\begin{abstract}
Background: Topical haemostatic agents are used to help achieve haemostasis during surgery when standard surgical techniques are insufficient. The objective of this study was to confirm the safety profile of an equine collagen patch coated with human fibrinogen and human thrombin with particular focus on the occurrence of thromboembolic events (TEEs), major bleeding and immunological events.
\end{abstract}

Methods: This was a non-interventional, multicentre, prospective, surveillance study in which a collagen fleece-bound fibrin sealant was prescribed in accordance with its marketing authorisation. The decision to use the sealant was based solely on current surgical practice. All patients that received the sealant and provided informed consent were included. TEEs (any coagula-based occlusion in a vessel or the heart identified by symptomatic clinical signs and/or verified by paraclinical examination), major bleeding (any bleeding that required intervention), and immunological events (hypersensitivity including anaphylaxis) that occurred during surgery, post-operative hospital stay or 6 months of followup were reported as adverse events. The primary endpoint was the proportion of patients experiencing a confirmed TEE.

Results: A total of 3098 patients were recruited at 227 centres in 12 European countries. The most frequent types of surgery were hepatic (33\%), gastrointestinal (16\%) and urological (14\%) and the main indication for surgery was for primary $(35 \%)$ or secondary $(20 \%)$ malignancy. Forty-six patients $(1.5 \%, 95 \% \mathrm{Cl} \mathrm{I.I-2.0 \% )} \mathrm{had} \mathrm{at} \mathrm{least} \mathrm{one} \mathrm{TEE} \mathrm{during}$ the study. The most commonly reported TEEs were pulmonary embolism or post-procedural pulmonary embolism $(\mathrm{n}=$ 18) and deep vein thrombosis $(n=9)$. There were 64 major bleedings in 62 patients and 9 immunological events in 8 patients.

Conclusion: Collagen fleece-bound fibrin sealant does not appear to be associated with an increased risk of TEEs, major bleeding or immunological events in patients undergoing surgery.

Trial registration: Clinicaltrials.gov number: NCT00285623 


\section{Background}

Rapid and effective control of bleeding during surgery reduces blood loss and can help decrease post-operative morbidity and mortality. Ligation, stapling, clipping and electrocautery are all widely used techniques to prevent bleeding. Over the past 20 years, a wide variety of topical haemostatic agents such as fleeces of different origin (collagen-based, e.g. Avitene ${ }^{\varpi}$; gelatine-based, e.g. Surgifoam ${ }^{\varpi}$, Gelfoam ; regenerated oxidised cellulose-based, e.g. Surgicel $^{\circledR}$, Curacel $^{\circledR}$ ), liquid fibrin sealants (e.g. Tisseel ${ }^{\circledR}$, Tissu$\mathrm{Col}^{\circledR}$, Evicel ${ }^{\circledast}$, Beriplast $\left.{ }^{\circledR}\right)$, albumin and glutaraldehyde bioglue $\left(\right.$ BioGlue $\left.^{\circledast}\right)$ and synthetic glues (e.g. CoSeal ${ }^{\circledast}$ ) have increasingly been used in a range of surgical procedures to help achieve haemostasis when standard surgical techniques are insufficient [1-3]. The use of these agents has a beneficial effect on surgical outcomes, including improved haemostasis, fewer complications and reduced duration of post-operative hospital stay $[1,2]$.

TachoSil ${ }^{\circledR}$ is a sterile, absorbable, haemostatic agent that consists of an equine collagen patch coated on one side with human fibrinogen and human thrombin. Unlike other fibrin sealants that require preparation before use, TachoSil is a ready-to-use fixed combination that is activated by moisture on application, providing adherence to the resection surface and haemostasis. The adhesive strength of TachoSil has been shown to be significantly higher than that of liquid fibrin glue [4] and the effect of the fibrinogen and thrombin together with the mechanical support of the collagen patch provides a physiologically extensible and pliable liquid and air tight seal [5]. TachoSil and its predecessor products, TachoComb ${ }^{\circledR}$ and TachoComb $\mathrm{H}$, have been used in a variety of surgical settings since being introduced in the early 1990s. TachoSil is indicated for supportive treatment in surgery for improvement of haemostasis, to promote tissue sealing, and for suture support in vascular surgery where standard techniques are insufficient.

Clinical studies have shown that TachoSil is effective in achieving haemostasis after kidney or liver resection $[6,7]$, as well as preventing air leakage after lung surgery $[8,9]$ and reducing lymphatic fluid production from the mediastinum [10]. TachoSil has also been shown to be safe and well tolerated, with occurrence of adverse events similar in TachoSil and non-TachoSil treated patients in controlled trials [6-10].

The objective of this study was to confirm the established safety profile of TachoSil with particular focus on the occurrence of thromboembolic events (TEEs), major bleeding and immunological events. The possible role of drug interactions in TEEs and major bleeding was also investigated.

\section{Methods}

This international, multicentre, prospective, surveillance study was of a non-interventional design, meaning that TachoSil was prescribed in accordance with the terms of its marketing authorisation. The decision to use TachoSil was made by the surgeon solely on the basis of current clinical practice. No additional diagnostic or monitoring procedures were applied.

Relevant Ethics Committees approved the protocol, and the trial was conducted in accordance with the Declaration of Helsinki, Good Pharmacoepidemiology Practice, the Data Protection Directive and any additional local regulations. A Data Monitoring Committee (DMC), which comprised of three surgeons (KG, DM, FM) not involved in recruiting patients to the study, was established before the protocol was finalised to regularly review the data. All patients provided written informed consent in accordance with local regulations, allowing the collection of personal data, direct data access and data processing. Consent could be provided either before or after surgery but all were obtained before data were entered into the study database. All patients that received TachoSil and provided informed consent were included in this study.

Reportable adverse events (AEs) were any TEE (a coagulabased occlusion in a vessel or the heart identified by symptomatic clinical signs and verified by paraclinical examination, e.g. ultrasound, magnetic resonance or computed tomography scan, or identified by paraclinical examination only [no routine paraclinical examination]), major bleeding (any bleeding that required intervention), or immunological event (hypersensitivity including anaphylaxis) that occurred during surgery, post-operative hospital stay or 6 months of follow-up. Follow-up at 6 months was done either by personal contact (telephone or visit) or by review of patients' medical records by the participating physician. Reportable AEs were coded by system organ class using MedDRA terminology (version 10.1). AE severity was defined as mild (transient symptoms, no interference with daily activities), moderate (marked symptoms, moderate interference with daily activities) or severe (considerable interference with daily activities). Serious AEs were defined as those that resulted in death, were life-threatening, required overnight inpatient hospitalisation or prolongation of existing hospitalisation, resulted in persistent or significant disability/ incapacity, involved congenital anomaly or birth defect, or that required intervention to prevent any of the previously listed. Causality between TachoSil and reportable AEs were assessed by the participating physician and defined as probable (good reason and sufficient documentation to assume a causal relationship), possible (causal relationship conceivable), unlikely or not related. 
If the reporting physician suspected that any thromboembolic or major bleeding event was possibly or probably caused by a drug interaction between TachoSil and a concomitant medication then this was documented.

Demographic characteristics, known risk factors for TEEs, bleeding and immunological events, ECG data, and surgical indication were documented for all patients. During surgery, the type and volume of blood products used was documented, as was the use of TachoSil. Use of anticoagulant therapy was assessed before, during and after surgery. Concomitant medication use was also recorded before, during and after surgery, as well as during the 6month follow-up.

\section{Statistical analysis}

The primary endpoint was the proportion of patients (with 95\% confidence intervals [CIs]) experiencing a confirmed TEE. All proportions were based on the number of patients exposed at a given timepoint with no adjustment for multiplicity or missing values. The number and percentage of patients experiencing a serious TEE or a TEE at least possibly related to TachoSil were also summarised.

Secondary endpoints included the proportion of patients experiencing an immunological event, the proportion of patients experiencing a major bleeding requiring interventional treatment, and drug interactions with TachoSil resulting in a TEE or major bleeding. Immunological events and major bleedings were summarised in a similar manner to TEEs.

A review of the literature available before the study began suggested that the incidence of TEEs for surgical procedures without the use of TachoSil was $10-15 \%$. On this basis, an observed incidence rate of $10 \%$ could be estimated with a precision (2-sided $95 \% \mathrm{CI}$ ) of $\pm 1.1 \%$ and an observed incidence rate of $15 \%$ could be estimated with a precision (2-sided $95 \% \mathrm{CI}$ ) of $\pm 1.3 \%$ with a sample size of 3000 patients.

During the study, all written consents were checked and collected data were verified against the source for approximately $10 \%$ of patients (patients with an ID number on their case report form with end digit 2, not revealed to sites until after the study) and for all reportable serious AEs.

\section{Results}

\section{Patient and surgical characteristics}

Between June 2005 and June 2007, 3098 patients received TachoSil while undergoing surgery and provided written informed consent to the use of their data. Patient data were collected from 227 surgical departments or clinics in 12 European countries (Germany, 1548 patients; France, $\mathrm{n}=461 ;$ Spain, $\mathrm{n}=343$; Greece, $\mathrm{n}=161 ;$ Austria, $\mathrm{n}=146$; the Netherlands, $\mathrm{n}=119$; Sweden, $\mathrm{n}=98$; Belgium, $\mathrm{n}=$ 82; Latvia, $\mathrm{n}=76$; $\mathrm{UK}, \mathrm{n}=41$; Norway, $\mathrm{n}=15$; and Denmark, $\mathrm{n}=8$ ). Almost all patient consents were obtained after surgery. No patients withdrew their consent during 6 months of follow-up. A total of 179 patients discontinued from the study early (death, $\mathrm{n}=156$; lost to follow-up, $\mathrm{n}$ = 20; other reasons, $\mathrm{n}=3$ ).

Over half of the patients were male (56\%), most were Caucasian $(98 \%)$ and their mean \pm SD age was $61 \pm 14$ years. The most frequent types of surgery were hepatic $(33 \%)$, gastrointestinal $(16 \%)$ and urological $(14 \%)$. The main indication for surgery was for primary (35\%) or secondary (20\%) malignancy. Ten percent of patients received surgery for benign neoplastic disease. Demographic characteristics and surgical variables are summarised in Table 1.

Of 2752 patients who had an ECG before surgery, 17.3\% ( $\mathrm{n}=477,15.4 \%$ of total study population) had abnormal results. Forty-three percent of patients $(n=1327)$ received anti-coagulant prophylaxis before surgery. Of these, 95\% had their coagulation status tested, and of those tested $11 \%(\mathrm{n}=139)$ had abnormal coagulation status. During surgery, or between surgery and hospital discharge, $80 \%$ of patients $(\mathrm{n}=2491)$ received anti-coagulant prophylaxis, with $20 \%(\mathrm{n}=440)$ of the $90 \%$ tested having abnormal coagulation status.

A total of 892 patients (29\%) received blood transfusion. Erythrocyte concentrate was the most frequently given blood product $(\mathrm{n}=736)$, followed by fresh frozen plasma $(\mathrm{n}=362)$, whole blood $(\mathrm{n}=84)$ and platelets $(\mathrm{n}=65)$. The median volume of blood products given was 3 units (range 1-83 units) (data available for 873 patients). The median area of TachoSil used was $45.6 \mathrm{~cm}^{2}$ (range 0.3$616.3 \mathrm{~cm}^{2}$ ), the size of one standard patch, based on the amount used during surgery without any correction for overlap of multiple patches.

The majority of patients (91\%) had at least one baseline risk factor for a TEE (Table 2). A risk factor for bleeding was reported for $8.2 \%$ of patients (bleeding or bleeding tendency, 4.6\%; abnormal pre-operative coagulation test, $4.5 \%$ ) and $15.3 \%$ of patients had a risk factor for an immunological event (known allergy, 13.4\%; any autoimmune disease, $2.6 \%$ ).

The most frequently used classes of concomitant medications were heparins (79\% of patients), proton pump inhibitors (60\%), anilides (e.g. paracetamol) (43\%), benzodiazepine derivatives (33\%), opioid anaesthetics (32\%), cephalosporins and related substances (31\%), 
Table I: Baseline demographic and surgical characteristics

\begin{tabular}{|c|c|c|c|c|}
\hline & All patients $(n=3098)$ & Patients with TEE $(n=46)$ & $\begin{array}{l}\text { Patients with major bleeding } \\
\qquad(\mathrm{n}=62)\end{array}$ & $\begin{array}{c}\text { Patients with immunological } \\
\text { event }(n=8)\end{array}$ \\
\hline Age (years), mean \pm SD & $60.7 \pm 14.0$ & $63.0 \pm 10.0$ & $58.6 \pm 16.5$ & $59.5 \pm 11.8$ \\
\hline Gender (female/male), \% & $43.7 / 56.3$ & $28.3 / 71.7$ & $32.3 / 67.7$ & $37.5 / 62.5$ \\
\hline BMI $\left(\mathrm{kg} / \mathrm{m}^{2}\right)$, mean $\pm \mathrm{SD}$ & $26.6 \pm 4.9$ & $27.2 \pm 4.4$ & $26.3 \pm 6.1$ & $26.0 \pm 5.4$ \\
\hline \multicolumn{5}{|l|}{ Type of surgery, n (\%): } \\
\hline Hepatic & 1007 (32.5\%) & $22(47.8 \%)$ & $16(25.8 \%)$ & $3(37.5 \%)$ \\
\hline Gastrointestinal & $500(16.1 \%)$ & $7(15.2 \%)$ & $13(21.0 \%)$ & $2(25.0 \%)$ \\
\hline Urological & $422(13.6 \%)$ & $2(4.3 \%)$ & $6(9.7 \%)$ & $\mathrm{I}(12.5 \%)$ \\
\hline Vascular & $227(7.3 \%)$ & $5(10.9 \%)$ & $3(4.8 \%)$ & 0 \\
\hline Pulmonary & $224(7.2 \%)$ & $4(8.7 \%)$ & $6(9.7 \%)$ & $\mathrm{I}(12.5 \%)$ \\
\hline Cardiac & $173(5.6 \%)$ & 0 & $5(8.1 \%)$ & $\mathrm{I}(12.5 \%)$ \\
\hline Gynaecological & $130(4.2 \%)$ & 0 & $\mathrm{I}(1.6 \%)$ & 0 \\
\hline Neurosurgical & $83(2.7 \%)$ & $2(4.3 \%)$ & I (I.6\%) & 0 \\
\hline Other & $424(13.7 \%)$ & $4(8.7 \%)$ & $10(16.1 \%)$ & 0 \\
\hline \multicolumn{5}{|l|}{ Indication for surgery, $\mathrm{n}(\%)$ : } \\
\hline Primary malignancy & $1074(34.7 \%)$ & $20(43.5 \%)$ & 17 (27.4\%) & $3(37.5 \%)$ \\
\hline Secondary malignancy & $619(20.0 \%)$ & $12(26.1 \%)$ & $7(\mid 1.3 \%)$ & $3(37.5 \%)$ \\
\hline Benign neoplastic disease & $306(9.9 \%)$ & $2(4.3 \%)$ & $7(11.3 \%)$ & 0 \\
\hline Other & 1098 (35.4\%) & $12(26.1 \%)$ & $31(50.0 \%)$ & $2(25.0 \%)$ \\
\hline Surgery, acute/elective, \% & $10.2 / 89.8$ & 10.9/89.1 & $24.2 / 75.8$ & $12.5 / 87.5$ \\
\hline $\begin{array}{l}\text { Duration of surgery (hours), } \\
\text { mean } \pm S D\end{array}$ & $3.2 \pm 1.8$ & $4.4 \pm 2.4$ & $3.7 \pm 2.4$ & $4.4 \pm 2.8$ \\
\hline $\begin{array}{l}\text { Patients receiving blood } \\
\text { transfusion, } \mathrm{n}(\%)\end{array}$ & $892(28.8 \%)$ & $14(30.4 \%)$ & $31(50.0 \%)$ & $2(25.0 \%)$ \\
\hline $\begin{array}{l}\text { Number of transfusion units } \\
\text { given, mean } \pm S D\end{array}$ & $5.2 \pm 7.3$ & $9.5 \pm 12.1$ & $10.0 \pm 15.9$ & $3.5 \pm 2.1$ \\
\hline $\begin{array}{l}\text { Area of TachoSil used }\left(\mathrm{cm}^{2}\right) \\
\text { mean } \pm \text { SD }\end{array}$ & $53.7 \pm 40.7$ & $60.1 \pm 41.4$ & $51.7 \pm 36.3$ & $55.1 \pm 40.8$ \\
\hline
\end{tabular}

plain sulphonamides (31\%), and other general anaesthetics $(28 \%)$.

\section{Safety}

A total of 124 AEs were reported; 51 TEEs in 46 patients, 64 major bleedings in 62 patients and 9 immunological events in 8 patients. Thirteen events were considered to be at least possibly related to TachoSil (3 TEEs, 8 major bleedings and 2 immunological events) by study surgeons or other physicians (Table 3 ).

\section{Thromboembolic events (TEEs)}

A total of 46 patients (1.5\%, 95\% CI 1.1-2.0\%) had at least one TEE at any time during the study (primary endpoint).
The most commonly reported TEEs were pulmonary embolism or post-procedural pulmonary embolism (18 patients), deep vein thrombosis $(n=9)$, phlebitis $(n=3)$, embolism (not otherwise specified) $(n=2)$, subclavian vein thrombosis $(n=2)$, portal vein thrombosis $(n=2)$, thrombosis (not otherwise specified) $(n=2)$ and myocardial infarction $(n=2)$. TEEs occurred on the day of surgery in 2 patients, and between the date of surgery and hospital discharge in 23 patients, with the remainder occurring during follow-up. TEEs by time of occurrence (up to one month post-surgery) are shown in Figure 1.

Twelve patients had mild TEEs (12 events), while moderate and severe TEEs each occurred in 17 patients (19 and 20 events, respectively). Forty-one patients had a TEE that was considered serious (46 events). 
Table 2: Patients with known risk factors at baseline for thromboembolic events

\begin{tabular}{|c|c|c|c|c|}
\hline Thromboembolic risk factor: & All patients $(n=3098)$ & Patients with TEE $(n=46)$ & Patients with no TEE $(n=3052)$ & P-value* \\
\hline Any thromboembolic risk factor & $2813(90.8 \%)$ & $42(91.3 \%)$ & $2771(90.8 \%)$ & $>0.99$ \\
\hline Cardiovascular (CV) risk factor: & $2135(68.9 \%)$ & $31(67.4 \%)$ & $2104(68.9 \%)$ & 0.87 \\
\hline Personal/family history of TEEs & $64(2.1 \%)$ & $2(4.3 \%)$ & $62(2.0 \%)$ & 0.25 \\
\hline$\geq \mathrm{I}$ other $\mathrm{CV}$ risk factor & $2122(68.5 \%)$ & $31(67.4 \%)$ & $2091(68.5 \%)$ & 0.87 \\
\hline Chronic obstructive pulmonary disease & $365(11.8 \%)$ & $6(13.0 \%)$ & $359(11.8 \%)$ & 0.82 \\
\hline Cancer & $1597(51.5 \%)$ & $30(65.2 \%)$ & $1567(5 \mid .3 \%)$ & 0.07 \\
\hline Metabolic/endocrinological risk factor & $797(25.7 \%)$ & $15(32.6 \%)$ & $782(25.6 \%)$ & 0.31 \\
\hline Other thromboembolic risk factor & $270(8.7 \%)$ & $6(13.0 \%)$ & $264(8.7 \%)$ & 0.29 \\
\hline Abnormal pre-operative ECG & 477 (15.4\%) & $10(21.7 \%)$ & $467(15.3 \%)$ & 0.22 \\
\hline
\end{tabular}

* From Fishers Exact test of $2 \times 2$ tables comparing for each risk factor to which extent the risk factor was predictive of the occurrence of TEEs

Three patients had a TEE that was considered at least possibly related to TachoSil by the study surgeon or other physician. Two of these were considered to be serious; one patient had phlebitis in the left leg 11 days after surgery, which was of moderate severity, while another patient had severe, post-procedural pulmonary embolism one week after surgery. Both patients recovered without sequelae. The third TEE considered possibly related to TachoSil was portal vein thrombosis of mild severity with onset two days after surgery. None of the seven TEEs leading to death were considered related to TachoSil.

\section{Major bleeding}

Sixty-two patients $(2.0 \%, 95 \% \mathrm{CI}: 1.5-2.6 \%)$ had at least one major bleeding (64 events). Most major bleedings occurred on the day of surgery $(n=24)$ or between surgery and hospital discharge $(\mathrm{n}=28)$ (Figure 1$)$.

A total of 55 patients had 57 serious major bleeding events, with 8 events in 7 patients considered at least possibly related to TachoSil. These included post-procedural haemorrhage in 3 patients, one on the day of surgery, one the day after surgery and one 4 days after surgery. One post-procedural haemorrhage was severe and 2 were moderate in severity. The other events considered at least possibly related to TachoSil were severe haemothorax, severe haemorrhage, moderately severe splenic haemorrhage, and moderately severe haematoma, all of which occurred on the day of surgery. In addition, drug ineffective was reported as an $\mathrm{AE}$ in the patient with moderately severe splenic haemorrhage. All 4 patients recovered, although the patient with haematoma died later from sepsis. None of the 8 major bleeding events leading to death was considered related to TachoSil.

\section{Immunological events}

Eight patients $(0.3 \%)$ experienced at least one immunological event. The most common immunological events were rash or rash pruritis, which occurred in 3 patients. No immunological events were reported during surgery, while 5 occurred between surgery and discharge. One immunological event was deemed serious but not related

Table 3: Occurrence of thromboembolic, major bleeding and immunological events

\begin{tabular}{lcccccc}
\hline & All & Mild & Moderate & Severe & Serious & TachoSil-related* \\
\hline Thromboembolic events & 51 & 12 & 19 & 20 & 46 & 3 \\
\hline Major bleeding & 64 & 4 & 27 & 33 & 57 & 8 \\
\hline Immunological events & 9 & 6 & 3 & 0 & 1 & 2 \\
\hline
\end{tabular}

*Considered at least possibly-related to TachoSil by the participating physician (probable = good reason and sufficient documentation to assume a causal relationship; possible = causal relationship conceivable). Mild = transient symptoms, no interference with daily activities; moderate = marked symptoms, moderate interference with daily activities; severe = considerable interference with daily activities; serious $=$ resulted in death, lifethreatening, required overnight inpatient hospitalisation or prolongation of existing hospitalisation, resulted in persistent or significant disability/ incapacity, involved congenital anomaly or birth defect, or that required intervention to prevent any of the previous occurrence of any of the previously listed. 


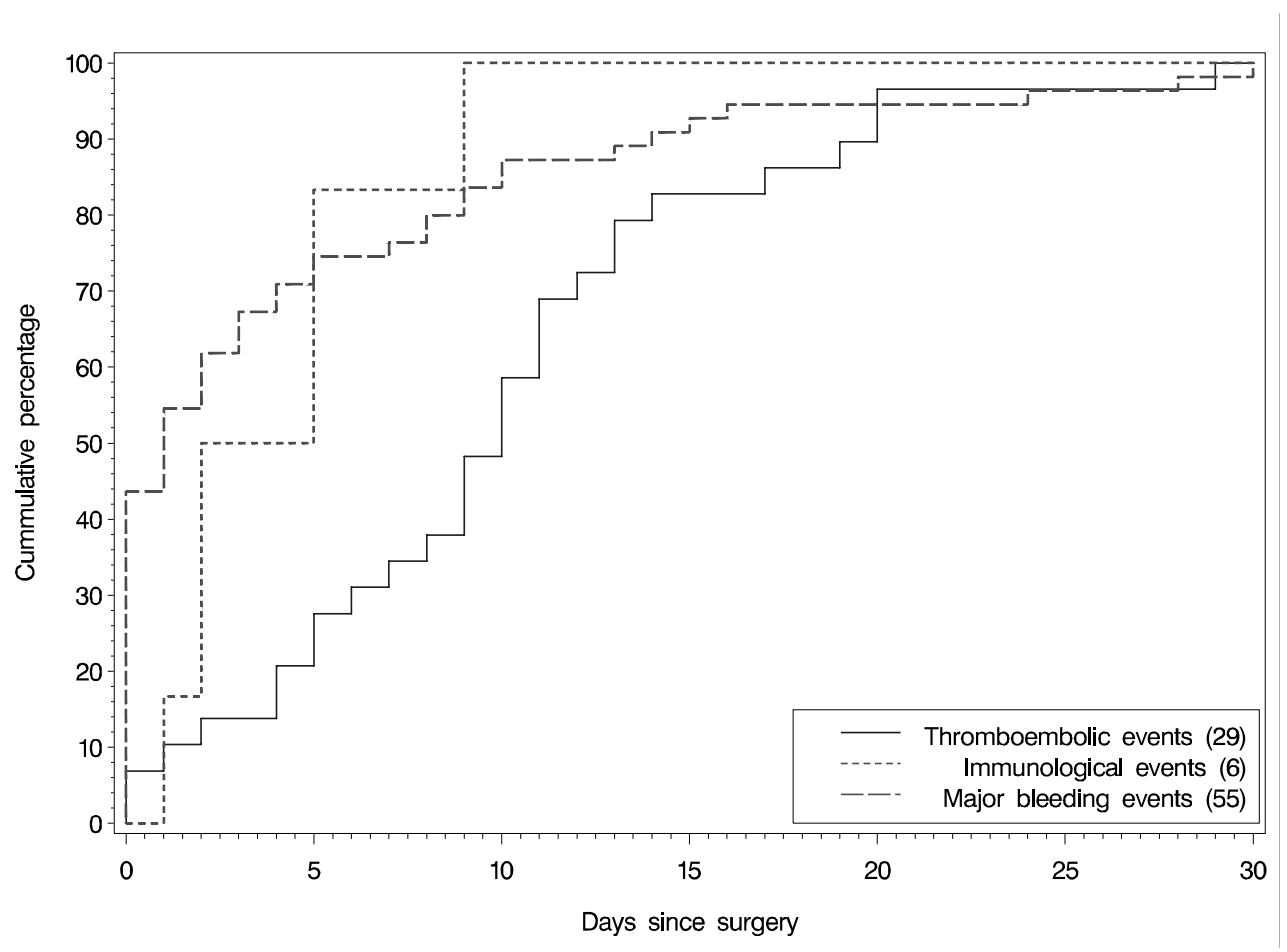

Figure I

Occurrence of thromboembolic, major bleeding and immunological events by time from surgery.

to TachoSil (increased white blood cell count). One patient had 2 immunological events considered to be at least possibly related to TachoSil (pyrexia and eosinophilia).

Previous exposure to TachoSil among patients was unknown. Seven patients were reported to be participating or had previously participated in another clinical trial or non-interventional study with TachoSil, while 2 patients received TachoSil on two occasions during this study. None of these patients experienced an immunological event.

\section{Drug interactions}

No drug interactions with TachoSil were suspected by the participating physician as the cause of a TEE or major bleeding. Over 38000 concomitant medications were recorded, coded and grouped to ATC code level 4 , with an average of 12.3 concomitant drugs per patient. To identify possible drug interactions, odds ratios (OR) were calculated post-hoc based on drug classes and number of exposed patients with and without events. With Hochberg correction for multiple testing, the only significant OR was for natural opium alkaloids and TEEs (OR 3.7, p = $0.022)$. However, this was considered to be attributable to the underlying conditions of patients receiving opioids, rather than any interaction with TachoSil.

\section{Discussion}

This prospective, non-interventional European surveillance study, conducted in a real-world clinical setting, suggests that patients undergoing surgery who receive TachoSil are not at increased risk of TEEs, major bleeding or immunological events.

The study cohort was representative of patients treated with TachoSil in clinical practice. The most common type of surgery was hepatic, which is the most documented use of TachoSil and was the most frequently observed type of surgery in a previous surveillance study [11]. Almost all patients $(94 \%)$ received TachoSil for haemostasis, with the other $6 \%$ receiving TachoSil for sealing purposes, such as the prevention of air leakage.

TachoSil did not appear to be associated with an increased risk of TEEs, with only three patients experiencing a TEE that was considered by the reporting physician to be at least possibly related to TachoSil. Possible causal relationships between TachoSil and AEs were not reviewed by the DMC. The overall reported incidence of TEEs in this study $(1.5 \%)$ is much lower than the expected $10-15 \%$, which was based on a review of the available literature. However, this expected incidence may have been an over-estimate. Meta-analyses of thromboprophylaxis in major surgery suggest that the incidence of TEEs in patients receiving 
unfractionated or low-molecular-weight heparin is only around 5\% [12-14]. Moreover, the incidence of clinically detected TEEs is only $1-2 \%$, given that the majority of TEEs are clinically silent and only detected using a systematic approach. Thus, the reported $1.5 \%$ occurrence in this study is consistent with previous observations, given that the majority of patients $(79 \%)$ received heparins during or after surgery and that no systematic testing for TEEs was done.

Under-reporting of reportable AEs is a concern in largescale non-interventional studies, since more events may go unnoticed and the recording of events in medical records may be less rigorously implemented compared with a controlled trial setting. In addition, events that are reported may be missed when data are transferred to the study due to inadequate staff training and monitoring and less intensive source data verification. In particular, the follow-up of patients transferred to other hospitals after surgery can be challenging and may lead to under-reporting of AEs. This was a particular concern in this study for those countries where 6-month follow-up could not be done through direct contact with patients, but only through review of medical records.

However, source data verification of $10 \%$ of patients did not suggest that a significant number of AEs were missed, with a similar occurrence of events reported for patients with different end digits of their patient number (Chisquared test, $p=0.8$ ). In addition, incidences of AEs were similar in patients followed-up by personal contact and those who only had their medical records reviewed. These findings suggest that under-reporting was not a significant problem in this study.

The reported 2\% incidence of major bleeding is in line with our experience. However, the protocol defined major bleeding as requiring interventional treatment. This definition may have been imprecise, especially given the heterogeneity of the cohort and range of surgical procedures. For instance, it is possible that major bleeding was considered in subjective terms, only being reported if in excess of what was expected. Transfusions were given to $29 \%$ of patients; however, transfusions are not only given because of a major bleeding, but may be given because of diseaserelated anaemia (e.g. in patients with cancer) or as a routine preventative measure for fluid and haemodynamic reposition, despite increasing evidence that this common clinical practice may be associated with increased morbidity and mortality [15].

Peri-operative anti-coagulant prophylaxis was reported in $80 \%$ of patients. The lack of anti-coagulant use in the remaining $20 \%$ may be largely attributable to underreporting of therapy. However, it is also possible that a trend towards earlier post-operative mobilisation of patients following surgery, at least in certain countries, may have reduced the use of prophylactic anti-coagulant therapy.

Another potential limitation with the design of this study was the issue of patient consent. All patients had to provide written informed consent, which could be obtained either before or after surgery. However, obtaining consent before surgery would have meant it was necessary to obtain consent from large numbers of patients who did not subsequently receive TachoSil, a considerable burden on the time and resources of participating centres. More importantly, obtaining consent before surgery carried the risk that surgeons may have been encouraged to use TachoSil in situations where they otherwise may not have done so. To avoid this, Ethics Committees in certain countries (Austria, Denmark and Germany) specified that consent must be obtained after surgery, in order to prove that surgeons' were not influenced in their decision to use TachoSil. However, obtaining consent after surgery meant that patients who died during surgery could not be included and AEs in these patients would not be reported, potentially resulting in selection bias and an underestimation of mortality and AEs. Illustrating this dilemma, six local Ethics Committees in Spain specified consent had to be obtained before surgery, while one stated that consent must be obtained post-surgery. In practice, almost all consents were obtained after surgery. However, since almost all reported TEEs occurred in the post-surgical phase, and since only $10 \%$ of TEEs and major bleedings had fatal consequences, the order of magnitude of any possible selection bias was likely to have been small.

Occurrence of immunological events was low $(<1 \%)$, suggesting there is no immunological risk with TachoSil. Hypersensitivity or allergic reactions may occur in rare cases in patients treated with fibrin sealant, especially if the preparation is applied repeatedly. Of particular concern, cross-reaction of antibodies against bovine thrombin and factor $\mathrm{V}$ with human factor $\mathrm{V}$ has been widely reported after exposure to topical bovine thrombin [16-18]. The risk of immunological reaction to TachoSil is minimal since the fibrinogen and thrombin are of human origin (no bovine material), while the equine collagen patch is free of immunogenic epitopes and is unlikely to induce an immune response [19]. It was impossible to ascertain whether patients had previous exposure to TachoSil. Seven patients reported that they had previously participated or were participating in another clinical study of TachoSil, although it was not possible to confirm whether these patients were actually treated with TachoSil. Two patients received TachoSil on two occasions during this study. None of these patients experienced an immunological event. 
Since the active ingredients of TachoSil are derived from human plasma, and the equine collagen fleece is derived from horse tendons, the potential for virus infection has to be considered. Standard measures to prevent infections resulting from the use of medicinal products prepared from human blood or plasma include selection of donors, and screening of individual donations and plasma pools for specific markers of infection. In addition, the manufacture of TachoSil includes several processing steps (e.g. pasteurisation, precipitation and adsorption, polymerasechain-reaction, sterilisation by gamma irradiation) intended to reduce the risk of viral transmission. No viral infections have been reported as AEs in clinical studies of TachoSil and no proven virus transmission has been detected up to the present since its approval in Europe.

There was no indication of any drug interactions between TachoSil and concomitant medications, as assessed by participating physicians and a post-hoc multiplicity corrected analysis of ORs of number of patients with and without events for each concomitant drug class.

\section{Conclusion}

In conclusion, TachoSil does not appear to be associated with an increased risk of TEEs, major bleeding or immunological events in patients undergoing surgery. In addition, there is no evidence of any interaction between TachoSil and other medications resulting in an increased risk of TEEs or major bleeding. These findings support previous experience that the use of TachoSil in a variety of surgical procedures is safe and well tolerated.

\section{Competing interests}

$\mathrm{MB}, \mathrm{JF}, \mathrm{SB}, \mathrm{KG}, \mathrm{DM}$ and FM declare they have no competing interests. TT is an employee of Nycomed.

\section{Authors' contributions}

KG, DM and FM comprised the Data Monitoring Committee, which provided input to the study protocol and was responsible for reviewing and evaluating study procedures and data. SB, MB and JF participated in the study and comprised the Publication Committee, which was responsible for preparation and review of the manuscript. TT was responsible for statistical analyses. All authors participated in the review, development and approval of the final manuscript.

\section{Acknowledgements}

Medical writing support was provided by Andy Bond of Spirit Medical Communications Ltd, supported by Nycomed.

This study and manuscript preparation was funded by Nycomed.

\section{References}

I. Bechstein WO, Strey C: Local and systemic hemostasis in surgery. Chirurg 2007, 78:95-100.
2. Mankad PS, Codispoti M: The role of fibrin sealants in hemostasis. Am J Surg 200I, I 82(2 SuppI):2IS-28S.

3. Seyednejad H, Imani M, Jamieson T, Seifalian AM: Topical haemostatic agents. $\mathrm{Br} /$ Surg 2008, 95: | | 97- I 225.

4. Erdogan D, de Graaf W, van Gulik TM: Adhesive strength of fibrinogen-coated collagen patch or liquid fibrin sealant in an experimental liver resection model in pigs. Eur Surg Res 2008, 41:298-302.

5. Carbon RT: Evaluation of biodegradable fleece-bound sealing: history, material science, and clinical application in tissue engineering and biodegradable equivalents. In Scientific and clinical applications Edited by: Lewandrowski K-U, Wise DL, Trantolo DJ, Gresser JD, Yaszemski MJ, Altobelli DE. Marcel Dekker; 2002:587-650.

6. Frilling A, Stavrou GA, Mischinger HJ, de Hemptinne B, Rokkjaer M, Klempnauer J, Thörne A, Gloor B, Beckebaum S, Ghaffar MF, Broelsch CE: Effectiveness of a new carrier-bound fibrin sealant versus argon beamer as haemostatic agent during liver resection: a randomised prospective trial. Langenbecks Arch Surg 2005, 390: II 4- I 20.

7. Siemer S, Lahme S, Altziebler S, Machtens S, Strohmaier W, Wechsel HW, Goebell P, Schmeller N, Oberneder R, Stolzenburg JU, Becker $\mathrm{H}$, Lüftenegger W, Tetens V, Van Poppel H: Efficacy and safety of TachoSil as haemostatic treatment versus standard suturing in kidney tumour resection: a randomised prospective study. Eur Urol 2007, 52: I | 56-I I63.

8. Lang G, Csekeö A, Stamatis G, LampI L, Hagman L, Marta GM, Mueller MR, Klepetko W: Efficacy and safety of topical application of human fibrinogen/thrombin-coated collagen patch (TachoComb) for treatment of air leakage after standard lobectomy. Eur J Cardiothorac Surg 2004, 25:160-166.

9. Anegg $U$, Lindenmann J, Matzi $V$, Smolle J, Maier A, Smolle-Jüttner F: Efficiency of fleece-bound sealing (TachoSil) of air leaks in lung surgery: a prospective randomised trial. Eur / Cardiothorac Surg 2007, 31 : 198-1202.

10. Czerny M, Fleck T, Salat A, Zimpfer D, Klepetko W, Wolner E, Mueller MR: Sealing of the mediastinum with a local hemostyptic agent reduces chest tube duration after complete mediastinal lymph node dissection for stage I and II non-small cell lung carcinoma. Ann Thorac Surg 2004, 77: 1028-1032.

11. Haas S: The use of a surgical patch coated with human coagulation factors in surgical routine: a multicenter postauthorization surveillance. Clin Appl Thromb Hemost 2006, 12:445-450.

12. Clagett GP, Reisch JS: Prevention of venous thromboembolism in general surgical patients. Results of meta-analysis. Ann Surg 1988, 208:227-240.

13. Mismetti P, Laporte S, Darmon JY, Buchmüller A, Decousus H: Metaanalysis of low molecular weight heparin in the prevention of venous thromboembolism in general surgery. Br J Surg 200I, 88:913-30.

14. Bottaro F], Elizondo MC, Doti C, Bruetman JE, Perez Moreno PD, Bullorsky EO, Ceresetto JM: Efficacy of extended thromboprophylaxis in major abdominal surgery: what does the evidence show? A meta-analysis. Thromb Haemost 2008, 99: II04-IIII.

15. Marik PE, Corwin HL: Efficacy of red blood cell transfusion in the critically ill: a systematic review of the literature. Crit Care Med 2008, 36:2667-2674.

16. Rapaport SI, Zivelin A, Minow RA, Hunter CS, Donnelly K: Clinical significance of antibodies to bovine and human thrombin and factor $\mathbf{V}$ after surgical use of bovine thrombin. Am J Clin Pathol 1992, 97:84-9|.

17. Berruyer M, Amiral J, Ffrench P, Belleville J, Bastien O, Clerc J, Kassir $A$, Estanove $S$, Dechavanne $M$ : Immunization by bovine thrombin used with fibrin glue during cardiovascular operations. Development of thrombin and factor $\mathbf{V}$ inhibitors. Thorac Cardiovasc Surg 1993, 105:892-897.

18. Streiff MB, Ness PM: Acquired FV inhibitors: a needless iatrogenic complication of bovine thrombin exposure. Transfusion 2002, 42: $18-26$

19. Adelmann-Grill BC, Otto K: Immunological safety evaluation of a haemostatic agent and wound dressing made of horse collagen fibrils. Arzneimittelforschung 1987, 37:802-805. 